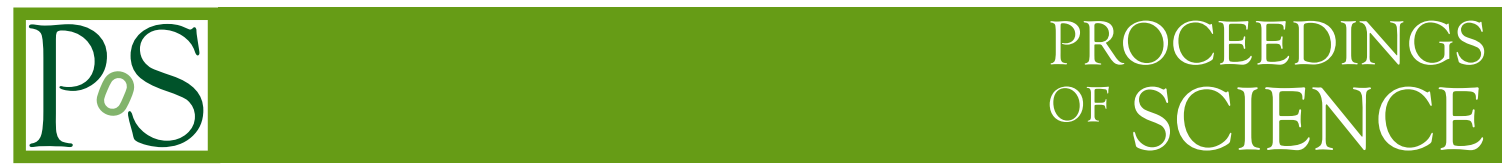

\title{
Causal Space-Time on a Null Lattice with Hypercubic Coordination
}

\author{
Martin Schaden* \\ Department of Physics, Rutgers University, Newark NJ 07102, USA \\ E-mail: mschaden@rutgers.edu
}

\begin{abstract}
I consider regularizing the first-order formulation of Hilbert-Palatini with cosmological constant by a causal null lattice with $S L(2, \mathbb{C})$ structure group. The lattice variables and invariant observables of the model are constructed and a condition that ensures that lattice configurations correspond to discretized causal manifolds is presented. An invariant regularization scheme is proposed and the $S L(2, \mathbb{C})$ structure group is localized to the compact structure group of spatial rotations.
\end{abstract}

The 32nd International Symposium on Lattice Field Theory,

23-28 June, 2014

Columbia University New York, NY

\footnotetext{
* Speaker.
} 


\section{Introduction}

It is appealing to quantize and regularize General Relativity (GR) by discretizing space-time. For Euclidean space-times, this approach was suggested by Regge (for a review, see [1]). The Causal Dynamical Triangulation (CDT) approach of [2] constructs causal manifolds only. This leads to greater stability and is rather promising. In CDT the basic simplexes are fixed, but the coordination number of vertices is variable. I here consider another approach that discretizes causal Lorentzian manifolds of indefinite metric by a lattice with fixed coordination but variable lightlike separations. It is more closely related to the continuum theory and the lattice integration measure essentially is dictated by the local $S L(2, \mathbb{C})$ structure group. A possible starting point is the continuum action of the first order Hilbert-Palatini formulation of GR with a cosmological constant,

$$
S_{\mathrm{HP}}=\frac{1}{l_{P}^{2}} \int_{M} e^{\alpha} \wedge e^{\beta} \wedge\left[\frac{\Lambda}{6} e^{\gamma} \wedge e^{\delta}-R^{\gamma \delta}(\omega)\right] \varepsilon_{\alpha \beta \gamma \delta},
$$

where the scale $l_{P}=\sqrt{32 \pi} \ell_{P}$ is proportional to the Planck length $\ell_{P}=\sqrt{G \hbar / c^{3}}$. Phenomenologically[3] the cosmological constant $\Lambda$ in natural units ${ }^{1}$, is rather small with $\lambda=\Lambda l_{P}^{2} \sim 6 \times 10^{-120}$.

The one-forms $e^{\alpha}$ in Eq.(1.1) are the Einstein-Cartan co-frames, $e^{\alpha}=e_{\mu}^{\alpha} d x^{\mu}$,

$$
\text { and } R^{\alpha \beta}(\omega) \text { is the } s o(3,1) \text { curvature two-form, } \quad R^{\alpha \beta}(\omega)=d \omega^{\alpha \beta}+\omega^{\alpha}{ }_{\gamma} \wedge \omega^{\gamma \beta}
$$

with connection $\omega^{\alpha \beta}=-\omega^{\beta \alpha}$. The Hilbert-Palatini action of Eq.(1.1) does not depend on the frame and is even defined if the co-frame is not everywhere invertible.

The formulation in terms of co-frames differs from one by frames in that it is polynomial in all fields and depends on the signed invariant volume element. The Lagrangian of Eq.(1.1) is proportional to $\operatorname{det}\left(e_{\mu}^{\gamma}\right)$ rather than $\left|\operatorname{det}\left(e_{\mu}^{\gamma}\right)\right|$ and changes sign under improper (local) Lorentz transformations. The action of Eq.(1.1) is classically equivalent to the Einstein-Hilbert action only for orientable manifolds with $\operatorname{det}\left(e_{\mu}^{\gamma}\right)>0$ everywhere. The remaining local internal symmetry of this oriented model is $S O(3,1)$ and causality restricts it to the connected component $S L(2, \mathbb{C})$.

Eq.(1.2) and Eq.(1.3) suggest to associate with each node $\mathbf{n} \in \boldsymbol{\Lambda}$ of a four dimensional hypercubic lattice $\boldsymbol{\Lambda}$ a local inertial system and a set of co-frames that represent the Minkowski displacements to neighboring events along each of four (forward) directions. As for an ordinary gauge theory, an $S L(2, \mathbb{C})$-matrix in the fundamental representation defines parallel transport (of a spinor) between two adjacent sites and is naturally associated with a lattice link.

Formulations such as this suffer from a number of drawbacks compared to purely geometrical ones, but enjoy some merits as well. Although not manifestly diffeomorphism invariant, it for instance is relatively straightforward to ensure causality by restricting to (forward) light-like displacements at each node: a configuration then is a set of consistent light-like displacements between causally connected nodes. We discuss some of the issues of such a formulation below and sketch solutions to some of them. Many interesting problems are as yet unsolved and will be considered elsewhere.

\footnotetext{
${ }^{1} \hbar=c=l_{P}=1$ in natural units and the Minkowski metric $\eta=\operatorname{diag}(1, \ldots, 1,-1)$.
} 


\section{Null lattice formulation}

We would like to construct a Lorentzian lattice model with metric signature $(+,+,+,-)$, an $S L(2, \mathbb{C})$ structure group and causal dynamics whose action in the classical limit $\hbar \rightarrow 0$ is given by Eq.(1.1) . The null-vector associated with an oriented link $[\mathbf{n}, \mu]$ of this lattice is represented by an anti- hermitian $2 \times 2$ matrix $E_{\mu}(\mathbf{n})$ that can be interpreted as the line integral,

$$
E_{\mu}(\mathbf{n})=l_{P}^{-1} \int_{\mathscr{C}(\mathbf{n}, \mu)} \sigma_{\alpha} e^{\alpha}=\sigma_{\alpha} E_{\mu}^{\alpha}(\mathbf{n})
$$

along the null geodesic $\mathscr{C}(\mathbf{n}, \mu)$ in forward direction from the event at $\mathbf{n}$ to the event $\mathbf{n}+\mu$ of the Lorentzian manifold. Here $\left\{e^{\alpha} ; \alpha=1, \ldots, 4\right\}$ is a local co-frame of one-forms and $\left\{\sigma_{\alpha} ; \alpha=\right.$ $1, \ldots, 4\}$ a fixed basis of anti-hermitian $2 \times 2$ matrices. The engineering dimension of the integral is absorbed by $l_{P}$ and all variables of the lattice model are dimensionless. The Planck length here is treated on the same footing as the fundamental constants $\hbar$ and $c$. The only dimensionless coupling is the cosmological constant $\lambda=\Lambda l_{P}^{2} \sim 6 \times 10^{-120}$. Contrary to ordinary field theories, the critical (UV) limit of the gravitational theory here is linked to its infra-red limit: as the UVcutoff is removed and the number of sites increased, $\lambda$ must be tuned so that the size of the universe described by the lattice approaches a finite limit in physical units of $l_{P}$. The cosmological constant in this sense can be viewed as a Lagrange multiplier for the average 4-volume of the universe.

Under local $S L(2, \mathbb{C})$ transformations, the $E_{\mu}(\mathbf{n})$ transform homogeneously,

$$
E_{\mu}(\mathbf{n}) \longrightarrow g(\mathbf{n}) E_{\mu}(\mathbf{n}) g^{\dagger}(\mathbf{n}) \text { with } g(\mathbf{n}) \in S L(2, \mathbb{C})
$$

On this space of (anti-)hermitian matrices, the $S L(2, \mathbb{C})$ invariant real scalar product is,

$$
A \cdot B:=-\frac{1}{2} \operatorname{Tr} \varepsilon A^{T} \varepsilon B=\frac{1}{2} \operatorname{Tr} \varepsilon A^{*} \varepsilon B \in \mathbb{R}
$$

where $X^{T}$ is the transpose and $X^{*}$ the complex conjugate of the (anti-)hermitian matrix $X . \varepsilon=$ $\left(\begin{array}{cc}0 & 1 \\ -1 & 0\end{array}\right)$ is an $S L(2, \mathbb{C})$ invariant. Note that the second form of the scalar product in Eq.(2.3) is invariant under $A, B \rightarrow g A g^{\dagger}, g B g^{\dagger}$ for all $g \in S L(2, \mathbb{C})$, regardless of whether $A$ and $B$ are antihermitian. Although no explicit choice[4] is required, we for convenience ${ }^{2}$ specify the basis of anti-hermitian $2 \times 2$ matrices $\left\{\sigma_{\alpha} ; \alpha=1,2,3,4\right\}$ to be,

$$
\sigma_{1}=\left(\begin{array}{cc}
0 & i \\
i & 0
\end{array}\right), \sigma_{2}=\left(\begin{array}{cc}
0 & -1 \\
1 & 0
\end{array}\right), \sigma_{3}=\left(\begin{array}{cc}
i & 0 \\
0 & -i
\end{array}\right), \sigma_{4}=\left(\begin{array}{ll}
i & 0 \\
0 & i
\end{array}\right) .
$$

\section{An Example: A null co-frame for Minkowski space}

That a causal manifold is described by linearly independent null vectors may surprise and it is reassuring to see this for ordinary flat Minkowski space.

A set of (forward) null co-frames that describes ordinary Minkowski space for instance is,

$e_{1}^{\mathrm{Mink}}=(-1,1,1, \sqrt{3}), e_{2}^{\mathrm{Mink}}=(1,-1,1, \sqrt{3}), e_{3}^{\mathrm{Mink}}=(1,1,-1, \sqrt{3}), e_{4}^{\mathrm{Mink}}=(-1,-1,-1, \sqrt{3})$.

\footnotetext{
${ }^{2}$ This basis distinguishes $\sigma_{4}$ from the three traceless, $\vec{\sigma}$, generators of the $s u(2)$-algebra $\left[\sigma_{\alpha}, \sigma_{\beta}\right]=2 \varepsilon(\alpha \beta \gamma 4) \sigma_{\gamma}$.
} 
These are the co-frames in a coordinate system $S^{\prime}$ related to ordinary Minkowski space-time with metric $\eta_{a b}=\operatorname{diag}(1,1,1,-1)$ by the coordinate transformation $4 x^{\prime 1}=-x^{1}+x^{2}+x^{3}+x^{4} / \sqrt{3}, 4 x^{\prime 2}=$ $x^{1}-x^{2}+x^{3}+x^{4} / \sqrt{3}, 4 x^{\prime 3}=x^{1}+x^{2}-x^{3}+x^{4} / \sqrt{3}, 4 x^{\prime 4}=-x^{1}-x^{2}-x^{3}+x^{4} / \sqrt{3}$. The transformation preserves orientation with $\operatorname{det}\left(e_{\mu}^{a}\right)=16 \sqrt{3}>0$. As required by Eq.(2.13) components of the metric in this coordinate system are negative semi-definite - in this case $g_{\mu \nu}=e_{\mu}^{a} \eta_{a b} e_{v}^{b}=-4$ for $\mu \neq v$, vanishing for $\mu=v$.

A Lorentz-transformed and scaled co-frames provides an equivalent descriptions of flat Minkowski space by a null-frame. Note that for flat space-time, lattice null-vectors are simply proportional to the co-frames $E_{\mu}^{\mathrm{Mink}}(\mathbf{n})=a \sigma_{\alpha} e_{\mu}^{\alpha \text { Mink }}$ with some global lattice constant $a$ that may be associated with the coarseness of the triangulation.

The anti-hermitian matrices $E_{\mu}(\mathbf{n})$ corresponding to null vectors in the forward light cone are singular. They may be represented[4] by complex bosonic 2-component $\operatorname{spinors}^{3} \xi_{\mu}^{A}, A=1,2$,

$$
E_{\mu}^{A \dot{B}}(\mathbf{n})=i\left(\xi_{\mu} \otimes \xi_{\mu}^{*}\right)^{A \dot{B}}=i \xi_{\mu}^{A}(\mathbf{n}) \xi_{\mu}^{* \dot{B}}(\mathbf{n}),
$$

where $\xi_{\mu}^{*}$ is the complex conjugate spinor. There is no summation over the repeated index ${ }^{4} \mu$ on the right-hand-side of Eq.(2.6). The (charge-)conjugate spinor $\chi^{c}$ is defined as, $\chi_{A}^{c}:=\chi^{B} \varepsilon_{B A}$. The spinor $\xi_{\mu}(\mathbf{n})$ and its conjugate $\xi_{\mu}^{c}(\mathbf{n})$ transform inversely under $S L(2, \mathbb{C})$,

$$
\xi_{\mu}^{A}(\mathbf{n}) \longrightarrow g_{B}^{A}(\mathbf{n}) \xi_{\mu}^{B}(\mathbf{n}) \Leftrightarrow \xi_{\mu A}^{c}(\mathbf{n}) \longrightarrow \xi_{\mu B}^{c}(\mathbf{n}) g_{A}^{-1 B}(\mathbf{n}), \text { for } g(\mathbf{n}) \in S L(2, \mathbb{C}) \text {. }
$$

A spinor may be compared to another by parallel transport along links of the lattice to a common node. This parallel transport is provided by matrices $U_{\mu}(\mathbf{n}) \equiv U[\mathbf{n}, \mu]$ in the fundamental representation of $S L(2, \mathbb{C})$. Every oriented link $[\mathbf{n}, \mu] \in \mathbf{\Lambda}$ thus is associated with a parallel transport matrix as well as a spinor. On a hypercubic null-lattice with $N$ nodes there are altogether $4 N$ spinors and $4 N$ parallel transport matrices. Under the $g(\mathbf{n}) \in S L(2, \mathbb{C})$ structure group, the transport matrices transform as,

$$
U_{\mu B}^{A}(\mathbf{n}) \rightarrow g_{C}^{A}(\mathbf{n}) U_{\mu D}^{C}(\mathbf{n}) g_{B}^{-1 D}(\mathbf{n}+\mu),
$$

and it is consistent with Eq.(2.8) to define,

$$
U\left[\mathbf{n}, \mathbf{n}^{\prime}\right]=U^{-1}\left[\mathbf{n}^{\prime}, \mathbf{n}\right] .
$$

Eqs. (2.7), (2.8) and (2.9) imply that $S L(2, \mathbb{C})$ invariants of this lattice model are (closed) loops of products of transport matrices and (open) strings of them bookended by spinors,

$$
\begin{aligned}
& C^{(r)}\left(\mathbf{n}_{1}, \mathbf{n}_{2}, \ldots, \mathbf{n}_{r}\right):=\operatorname{Tr} U\left[\mathbf{n}_{1}, \mathbf{n}_{2}\right] U\left[\mathbf{n}_{2}, \mathbf{n}_{3}\right] \ldots U\left[\mathbf{n}_{r}, \mathbf{n}_{1}\right], \\
& O_{\mu \nu}^{(r)}\left(\mathbf{n}_{0}, \mathbf{n}_{1}, \ldots, \mathbf{n}_{r}\right):=\xi_{\mu}^{c}\left(\mathbf{n}_{0}\right) U\left[\mathbf{n}_{0}, \mathbf{n}_{1}\right] U\left[\mathbf{n}_{1}, \mathbf{n}_{2}\right] \ldots U\left[\mathbf{n}_{r-1}, \mathbf{n}_{r}\right] \xi_{v}\left(\mathbf{n}_{r}\right) .
\end{aligned}
$$

Observables are sums of products of these invariants and of their complex conjugates. The lattice action correspondiong to Eq.(1.1) may be constructed from the short invariants,

$$
\begin{aligned}
f_{\mu v}(\mathbf{n}) & :=\xi_{\mu A}^{c}(\mathbf{n}) \xi_{v}^{A}(\mathbf{n})=\xi_{\mu}^{A}(\mathbf{n}) \varepsilon_{A B} \xi_{v}^{B}(\mathbf{n})=: O_{\mu v}^{(0)}(\mathbf{n}), \\
\mathscr{V}_{\mu \nu \rho \sigma}(\mathbf{n}) & :=-i f_{\mu v}^{*}(\mathbf{n}) f_{v \rho}(\mathbf{n}) f_{\rho \sigma}^{*}(\mathbf{n}) f_{\sigma \mu}(\mathbf{n}), \\
\chi_{\mu v \rho \sigma}(\mathbf{n}) & :=\xi_{\mu A}^{c}(\mathbf{n}) U_{\rho B}^{A}(\mathbf{n}) U_{\sigma C}^{B}(\mathbf{n}+\rho) \xi_{v}^{C}(\mathbf{n}+\rho+\sigma)=: O_{\mu v}^{(2)}(\mathbf{n}, \mathbf{n}+\rho, \mathbf{n}+\rho+\sigma) .
\end{aligned}
$$

\footnotetext{
${ }^{3} E_{\mu}^{A \dot{B}}(\mathbf{n})$ is a Lorentz vector that transforms as an $\operatorname{sl}(2, \mathbb{C})$ tensor in the $\left(\frac{1}{2}, \frac{1}{2}\right)$ representation.
}

${ }^{4}$ Only diagonally related repeated indices, i.e. ${ }_{A} \nearrow^{A}$ and ${ }^{B} \searrow_{B}$, are automatically summed over. 
The 6 complex quantities $f_{\mu v}(\mathbf{n})=-f_{v \mu}(\mathbf{n})$ are constrained by,

$$
\operatorname{Pf}(f(\mathbf{n}))=f_{12}(\mathbf{n}) f_{34}(\mathbf{n})+f_{13}(\mathbf{n}) f_{42}(\mathbf{n})+f_{14}(\mathbf{n}) f_{23}(\mathbf{n})=0 .
$$

$f_{\mu v}(\mathbf{n})$ thus is given by only 10 real parameters. Four are overall phases of the spinors[4] on which the metric does not depend (see Eq.(2.15) below). The remaining 6 are the spatial lengths,

$$
\ell_{\mu \nu}^{2}(\mathbf{n})=-2 E_{\mu}(\mathbf{n}) \cdot E_{v}(\mathbf{n})=\operatorname{Tr} \varepsilon E_{\mu}^{T}(\mathbf{n}) \varepsilon E_{v}(\mathbf{n})=\left|f_{\mu \nu}(\mathbf{n})\right|^{2} \geq 0,
$$

where Eqs. (2.6) and (2.11a) have been used. The components of the metric tensor in this coordinate system thus are negative semi-definite and satisfy triangle inequalities. The (signed) local invariant 4-volume is given by,

$$
V(\mathbf{n})=\frac{1}{48} \sum_{\mu \nu \rho \sigma} \varepsilon(\mu v \rho \sigma) \mathscr{V}_{\mu v \rho \sigma}(\mathbf{n})=\operatorname{det} E_{\mu}^{\alpha}(\mathbf{n}),
$$

and the metric is singular only if one of its off-diagonal components vanishes.

Requiring invariance under local $U(1)$ phase transformations of the spinors,

$$
\xi_{\mu}(\mathbf{n}) \rightarrow e^{i \phi_{\mu}(\mathbf{n})} \xi_{\mu}(\mathbf{n}) \text { and } \xi_{\mu}^{*}(\mathbf{n}) \rightarrow e^{-i \phi_{\mu}(\mathbf{n})} \xi_{\mu}^{*}(\mathbf{n}),
$$

further constrains physical observables to locally conserve four separate spinor numbers. Physical observables with vanishing local spinor numbers depend only on the combination of spinors that form the anti-hermitian co-frames of Eq.(2.6). There is no obvious reason for requiring this Abelian gauge symmetry. It could be accidental and emerge at low energies only. However, this symmetry appears to play an essential rôle in constraining lattice configurations to ones that are triangulations of manifolds.

\section{The Manifold condition}

While it may be plausible that any causal Lorentz manifold may be triangulated by a nulllattice, it is equally clear that a given configuration of spinors in general will not describe a triangulated manifold. This is for instance evident if one considers the null-frames of Minkowski space of Eq.(2.5) with a site-dependent proportionality constant. However, one can derive conditions for a spinor configuration to represent a triangulated causal Lorentz manifold. Somewhat surprisingly I find that any (oriented) spinor configuration that satisfies,

$$
\begin{aligned}
\operatorname{Pf}[\widetilde{f}(\mathbf{n})] & =0 \text { and } V(\mathbf{n})>0, \forall \mathbf{n} \in \boldsymbol{\Lambda}, \text { where } \\
\widetilde{f}_{\mu v}(\mathbf{n}) & :=f_{\mu v}(\mathbf{n}-\mu-v)=\xi_{\mu}^{A}(\mathbf{n}-\mu-v) \varepsilon_{A B} \xi_{v}^{B}(\mathbf{n}-\mu-v),
\end{aligned}
$$

appears to describe a causal manifold. The converse also seems to hold: any (oriented) causal manifold can be triangulated by a spinor configuration that satisfies Eq.(3.1). Although $S L(2, \mathbb{C})$ invariant, Eq.(3.1) is not invariant under the local Abelian $U(1)^{4}$ symmetries and the proper statement is that a set of spinor phases can be found so that (3.1) holds, only if the corresponding configuration of null-vectors describes a discretized causal manifold. Eq.(3.1) evidently is a crucial ingredient of the formulation, but is still under investigation and a detailed proof will only be presented elsewhere. 


\section{Regularization}

We so far have not regularized the triangulation of a causal Lorentz manifold. Nothing prevents the model from describing an arbitrary 'small' portion of the manifold with an exorbitant number of nodes. To construct a sensible continuum limit, a lattice cutoff that governs the coarseness of the triangulation is required. I suggest to use a cutoff that depends on the signed local 4-volume of the lattice at site $\mathbf{n}$ defined in (2.14), i.e. by for instance requiring that,

$$
V[\mathbf{n}] \geq \varepsilon^{4}>0, \forall \mathbf{n} \in \Lambda .
$$

Here the dimensionful length $a=\varepsilon l_{P}$. Eq.(4.1) not only regulates the model in a diffeomorphism invariant fashion, but simultaneously eliminates the notorious sign problem of the HP-action. Of course, exponential or other "softer" cutoffs may be used instead. This modification of the local lattice integration measure should allow one to study the limit $\varepsilon^{4} \rightarrow 0$ while adjusting the number of sites $N$ and the dimensionless coupling $\lambda(e p s, N)$ of the theory. Whether such a critical limit exists, remains to be seen.

\section{Localization of $S L(2, \mathbb{C})$ to the compact structure group $\mathrm{SU}(2)$}

Another peculiar feature of a lattice theory of this type not found in ordinary LGT's is that its $S L(2, \mathbb{C})$ structure group is not compact. Although the (infinite) volume of this structure group still formally cancels in expectation values of $S L(2, \mathbb{C})$-invariant observables, one is formally dividing infinite factors. This is bound to be a rather unstable procedure and it seems advisable to partially localize $S L(2, \mathbb{C})$ to a compact subgroup and arrive at a well-defined (finite) lattice measure.

The partial "gauge fixing" corresponds to choosing local inertial systems at each node with certain (physical) characteristics. We propose to localize to the compact $S U(2) \subset S L(2, \mathbb{C})$ rotation group of local Lorentz transformations.

Consider the local $S U(2)$-invariant Morse function constructed from the $S L(2, \mathbb{C})$-transformed spinors of a site,

$$
M_{\xi}[g(\mathbf{n})]=\sum_{\mu} \xi_{\mu}^{\dagger}(\mathbf{n}) g^{\dagger}(\mathbf{n}) g(\mathbf{n}) \xi_{\mu}(\mathbf{n})=\sum_{\mu} \tau_{\mu}^{(g)}(\mathbf{n}) .
$$

which is the sum of the positive time components $\tau_{\mu}^{(g)}$ of the $S L(2, \mathbb{C})$-transformed null vectors. Considered as a function of the $S L(2, \mathbb{C})$-transformation, $g(\mathbf{n})$ the Morse function of Eq.(5.1) clearly is a function of $S L(2, \mathbb{C}) / S U(2)$ only and is bounded below. Decomposing $g \in S L(2, \mathbb{C})$ in a hermitian and a unitary component, both of unit determinant, $g=u h$, with $h=h^{\dagger}=\exp -i \vec{v} \cdot \vec{\sigma}, u \in$ $S U(2)$, extrema of the Morse function $M$ are characterized by,

$$
0=-i \sum_{\mu} \xi_{\mu}^{\dagger}(\mathbf{n}) \vec{\sigma} \xi_{\mu}(\mathbf{n})
$$

The solution of (5.2) is unique modulo $S U(2)$, because the Hessian matrix $H$,

$$
H_{i j}(\mathbf{n})=\delta_{i j} \sum_{\mu} \tau_{\mu}(\mathbf{n}),
$$

is stictly positive. It vanishes only if $\sum_{\mu} \tau_{\mu}=0$, that is when all null-vectors of a node vanish. The solution to Eq.(5.2) therefore is the unique absolute minimum of the Morse potential (modulo 
local spatial rotations). The constraint Eq.(5.2) may be implemented by local Lagrange multipliers or can be used to eliminate the dependence on one of the four null vectors at each site. Either way, the Hessian of Eq.(5.3) is positive and $\left(\operatorname{det}[H]=\sum_{\mu} \tau_{\mu}(\mathbf{n})\right)^{3}$ may be included in the local measure.

\section{Discussion and some more speculation}

We considered the construction of a hypercubic null lattice with $S L(2, \mathbb{C})$ structure group. In this model the light-like displacements between adjacent nodes are associated with spinors and $S L(2, \mathbb{C})$ transport matrices. The coordination of the lattice itself is hypercubic but is used only for labeling events. Any spinor configuration that satisfies the constraints of Eq.(3.1) appears to correspond to a causal manifold. The $S L(2, \mathbb{C})$-invariant observables of this theory were constructed and an invariant UV-regularization of the model was suggested in Eq.(4.1). The non-compact $S L(2, \mathbb{C})$ structure group of this model was localized to the compact $S U(2)$ subgroup of spatial rotations by a Morse construction. The Hessian of this localization is positive definite and unique (modulo $S U(2)$ ), and does not suffer a Gribov problem. The description of Minkowski space by a co-frame of null vectors in Eq.(2.5) incidentally satisfies the gauge condition of Eq.(5.2). The most important issue not touched in this article, is whether the formulation admits a critical continuum limit.

The advantage of such a formulation may be that invariance under $S L(2, \mathbb{C})$ severely constrains the lattice integration measure. It essentially is uniquely determined by the transformation properties of the field content. Without continuation to Euclidean space the HP-action is purely imaginary and not suitable for conventional Monte-Carlo simulations. However, complex Langevin equations[5] are a promising tool for numerically investigating such models.

\section{References}

[1] R. Loll, Living Rev.Rel. 1, 13 (1998), arXiv:gr-qc/9805049 [gr-qc] ; A. P. Gentle, Gen.Rel.Grav. 34, 1701 (2002), arXiv:gr-qc/0408006 [gr-qc] .

[2] J. Ambjorn, J. Jurkiewicz, and R. Loll, , 321 (2010), arXiv:1004.0352 [hep-th] ; J. Ambjorn, A. Görlich, J. Jurkiewicz, and R. Loll, Int.J.Mod.Phys. D22, 1330019 (2013).

[3] J. D. Barrow and D. J. Shaw, Gen.Rel.Grav. 43, 2555 (2011), arXiv:1105.3105 [gr-qc] .

[4] R. Penrose and M. A. MacCallum, Phys.Rept. 6, 241 (1972).

[5] G. Aarts, F. Attanasio, B. Jäger, E. Seiler, D. Sexty, et al., PoS LATTICE2014, 200 (2014), arXiv:1411.2632 [hep-lat] . 